\title{
Rate Loss Due to Power Equalization in Cellular Communications
}

\author{
Bixio Rimoldi ${ }^{b}$ Long Duan ${ }^{b b}$ and Quinn Libbb \\ ${ }^{b}$ EPFL, Mobile Communications Laboratory, CH-1015 Lausanne \\ bixio.rimoldi@epfl.ch \\ ${ }^{b b}$ Ericsson Wireless Communications, San Diego, California \\ l.duan@ericsson.com \\ ${ }^{b b b}$ Lucent Technology, Whippany, New Jersy \\ quinnli@lucent.com
}

March 9, 2001

\begin{abstract}
For a cellular communication network, the loss in up-link bandwidth-efficiency resulting from power equalization is considered. Here power equalization refers to the operation of adjusting the transmit power of mobile units in such a way that the received power is the same for all units whereas bandwidth-efficiency is measured in terms of sum-rate per cell divided by the total system bandwidth. A single-cell system is considered first, and the bandwidth efficiency is determined with and without power equalization as well as with and without shadowing. The analysis is then extended to multi-cell-systems. It is found that in all cases power equalization severely penalizes the bandwidth-efficiency.
\end{abstract}

\section{Introduction}

The main motivation for this paper is to investigate the effects of power equalization in up-link cellular communications, where by power equalization we mean the action taken by mobile transmitters to ensure that all signals are received with the same intensity. ${ }^{1}$ We find that power-equalization severely limits the maximal possible sum-rate.

We come to this conclusion by looking at the maximal sum-rate achievable under various scenarios, including a multi-cell system in which shadowing is taken into account. The maximal sum-rate is the

\footnotetext{
${ }^{1}$ We introduce the term power equalization as opposed to power control since the latter is also widely used to refer to the action of controlling the power at the transmitter so as to optimize some objective function such as the mutual information of the resulting channel.
} 
average mutual information between all channel inputs and the channel output. In the case of fading we assume that the fading state is known to the receiver. In all cases, we find that having the transmitpower uniformly distributed over each cell brings a significant increase in sum-rate with respect to a system which implements power equalization. ${ }^{2}$ The sum-rate for the the various situations described in this paragraph is plotted in Fig. 6.

The assumption that the fading state (when applicable) is known at the receiver is acceptable when the channel does not change rapidly. For instance in a conference room where a number of laptops are transmitting to a base station in time-division duplex (TDD) mode one can assume that the channel state is known to the receiver and that it does not vary significantly over the time spanned by a codeword. Then, at any given time, one can model the channel as the well-understood Gaussian multiple-access channel. One can approach any point in the capacity region of a Gaussian multiple access channel by means of powerful codes for (point-to-point) additive Gaussian channels and successive decoding [1]. Low-density parity-check codes [4, 5, 3] and turbo codes [2] are the most promising family of codes for this purpose. This justifies the position that we are taking in this paper, namely that we essentially know how to approach any point in the capacity region of the channels considered in this paper.

It remains to justify using the maximal sum-rate to evaluate the impact of power equalization. Since we essentially know how to approach any point in the capacity region, it seems natural to ask how to compare competing capacity regions. We don't know of any trivial answer to this question. In the past, several authors have compared capacity regions by means of their symmetric capacity. For an $M$-user channel the symmetric capacity is defined as the supermum of the rate $R$ such that $(R, R, \ldots, R) \in$ $\mathbf{R}^{M}$ is in the capacity region. The symmetric capacity is a reasonable performance measure if all transimtters request the same rate. This equal-rate assumption is valid for voice communication but it will be less and less a valid assumption in the future as we move toward integrated systems. Such systems will comprise a broad spectrum of terminal equipments, some of which capable of varying their transmit power and/or transmission rate over several orders of magnitude. Applications will request transmission rates varying over the whole range of possibilities supported by the terminal equipments. Vis á vis of these considerations, the maximal sum-rate seems to be a more suitable

\footnotetext{
${ }^{2}$ One can alwasy find a suboptimal multiple-access technique for which power equalization is beneficial. For instance this is the case for spread spectrum multipl access (SSMA) with conventional matched-filter detectors since in this case power equalization is needed to combat the so-called near-far problem.
} 
performance descriptor than the symmetric capacity. It describes the position of the most interesting subregion of the capacity region, namely the dominant face (see e.g. [1]), rather than describing a single point. Moreover, the maximal sum-rate is easier to compute since it requires evaluating a single mutual information.

The assumptions that we are making about the channel are not always justifiable in practice. In particular, in many cases of interest the receiver does not know the channel state. We believe that focussing only on the most difficult scenarios, e.g. on the case of unknown channel state at the receiver, is too of a conservative approach. Different scenarios require different channel models and different communications techniques. Hopefully one day we will understand all major scenarios, we will know what to do in each case, and software-defined radio will allow us to implement the best strategy for each scenario of interest. In this paper we consider one of the most favorable scenarios, namely when the channel state is known to the receiver.

Even though successive decoding is not the focus of this paper, it should be pointed out that it is a technique of fundamental importance which allows one to approach any rate in the capacity region of channels that are much more general than those considered in this paper. In particular, successive decoding does not require that the channel be additive and that the fading state be known at the receiver. The generality of successive decoding lies in the fact that it is a procedure suggested by the chain rule of mutual information. To the contrary of a widespread belief, successive decoding does not just mean decoding a user, estimating its contribution at the receiver, and subtracting its contribution from the received signal (which makes sense only for additive channels when the fading state is known at the receiver). To learn more about successive decoding in relationship to achieving any point in the capacity region the interested reader is referred to [1] for the Gaussian case, to [6] for a discussion of the fading case, to [7] for general memoryless multiple-access channels, and to [8] for fading channels with fading state known at transmitter and receiver). A discussion of successive decoding limited to the vertices of the capacity region may be found in the excellent textbook [9, p.396].

This paper should clarify two misconceptions. The first misconception is that power equalization is beneficial in cellular communications in general. Power equalization is needed to combat the socalled near-far problem of direct-sequence spread-spectrum multiple-access (SSMA) (see e.g. the IS-95 standard) with matched-filter detectors. Possibly as a consequence of the success of IS-95, many 
believe that power equalization is a beneficial feature in general. ${ }^{3}$

The second misconception is a consequence of the first. It is sometimes claimed that successive decoding does not bring much if one considers a cell cluster as opposed to a single cell in isolation. For instance in [10] it is shown that successive decoding (called interference cancellation) does not significantly increase the sum-rate with respect to the simpler technique of decoding each user independently, considering all the other users as noise. This conclusion in [10] is only valid in the presence of power equalization. Our paper, which generalizes [11] to account for shadowing effects, shows that successive decoding (or for that matter any decoder which allows one to approach the maximal sum-rate) brings a significant gain when we do not equalize powers (see Fig. 6).

The paper is organized as follows. In Section 2 we consider a single cell in isolation and compute and compare bandwidth efficiencies with and without power equalization. The bandwidth efficiency is defined as the sum-rate per cell divided by the system bandwidth. For the system without power equalization we compute the bandwidth efficiency with and without considering shadowing effects. In Section 3 we extend the results of Section 2 to multi-cell systems. Section 4 contains concluding remarks.

\section{Single-Cell System}

We first evaluate the bandwidth efficiency for an isolated cell with and without power equalization. The latter is studied with and without shadowing. (Power equalization and shadowing are not considered as a separate case the former elimnates the effect of the latter.)

The cell is depicted in Figure 1 with a base station at its center. Assume that there are $M$ transmitters scattered in the shaded region $A$ of area $|A|$. The inner and outer radii of the region of interest are $R_{i}$ and $R_{o}$, respectively.

\subsection{With Power Equalization}

Power equalization is typically used to eliminate the well-known near-far problem associated to spreadspectrum multiple-access (SSMA) (see e.g. [12], [13]). The output power of each transmitter is adjusted so that all signals are received at the base station with the same power.

Let $C$ denote the maximum sum-rate that can be achieved by a multiple-access system. Let $W$

\footnotetext{
${ }^{3}$ Some sort of power control may be needed due to the limitations of today's electronic, but this is a different story.
} 


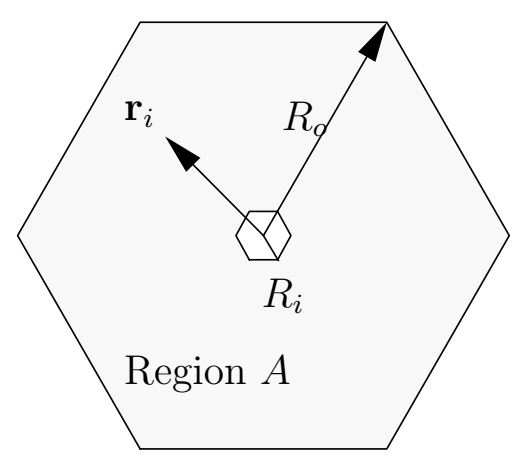

Figure 1: A single-cell system.

be the system bandwidth. We define the bandwidth efficiency of the system as the ratio $C / W$. The bandwidth efficiency of a single-cell system with power equalization can be derived as follows. Let $P L$ be the received power for a single user where $P$ is the transmit power and $L$ the propagation loss. Due to power equalization, each one of the $M$ users in the cell will be received with the same power $P L$. Hence, the total received power at the base station is

$$
P_{T_{c}}=M P L
$$

When shadowing effects are not considered, a mobile experiences the largest propagation loss at the cell boundary. Assuming the standard fourth power propagation loss [12], we have $L=G / R_{o}^{4}$, where $G$ is a constant antenna gain. Hence,

$$
P_{T_{c}}=\frac{M G P}{R_{o}^{4}}
$$

Given the total received power $P_{T_{c}}$, the largest possible bandwidth efficiency for a system that equalizes powers is given by [9, Section 14.3.6]

$$
\frac{C}{W}=\log \left(1+\frac{P_{T_{c}}}{N_{0} W}\right)[\text { bits } / \mathrm{s} / \mathrm{Hz}],
$$

where $N_{0}$ is the single-sided power spectrum density of the background noise. As pointed out in the introduction, the bandwidth efficiency in (3) can be achieved with interference cancellation. This is true regardless of individual rates, provided that the rate tuple $R=\left(R_{1}, \ldots, R_{M}\right)$ lies inside the capacity region.

For the single-cell SSMA system that decodes each user while treating all other users as noise, the noise seen by each user is $(M-1) P L+N_{0} W$. The bandwidth efficiency is then given by [11]

$$
\frac{C}{W}=M \log \left(1+\frac{P L}{(M-1) P L+N_{0} W}\right)[\mathrm{bits} / \mathrm{s} / \mathrm{Hz}] .
$$


The right side of both equation (3) and (4) increase monotonically with $M$. However, as shown in Figure 2, the former increases without bound whereas the latter saturates at

$$
\lim _{M \rightarrow \infty} \frac{C}{W}=\lim _{M \rightarrow \infty} M \log \left(1+\frac{P L}{(M-1) P L+N_{0} W}\right)=\log e[\mathrm{bits} / \mathrm{s} / \mathrm{Hz}] .
$$

\subsection{Without Power Equalization And Without Shadowing}

Power equalization is necessary for SSMA systems with matched filter detectors but it is not a fundamental requirement. As mentioned earlier, power equalization is not necessary for a successive decoder. For the rest of this section we assume that each transmitter has the same output power $P$.

Let user $i, 0<i \leq M$, be at some location $\mathbf{r}_{i}$ in the shaded region of Figure 1 . We assume that $\mathbf{r}_{1}, \ldots \mathbf{r}_{M}$, are mutually independent random variables with probability density function $p_{i}(\mathbf{r})$ on $A$. Then, given $A_{0} \in A$,

$$
\operatorname{Pr}\left\{\mathbf{r}_{i} \in A_{0}\right\}=\int_{A_{0}} p_{i}(\mathbf{r}) \mathrm{d} \mathbf{r} .
$$

Let $r\left(\mathbf{r}_{i}\right)$ denote the distance from user $i$ to his base station. Assuming fourth order path loss (and no shadowing), the path loss of user $i$ is proportional to $\left(r\left(\mathbf{r}_{i}\right)\right)^{-4}$. To model the fact that user $i$ does not transmit all the time, we use a random variable $\psi_{i} \in\{0,1\}$, with $\alpha_{i} \triangleq \operatorname{Pr}\left\{\psi_{i}=1\right\}$, where $\psi_{i}=1$ signifies that user $i$ is transmitting.

The power received at the base station from user $i$ at location $\mathbf{r}_{i}$ is given by

$$
P_{i}=G P \psi_{i}\left(r\left(\mathbf{r}_{i}\right)\right)^{-4}
$$

The sum power at the base station is given by

$$
P_{T}=\sum_{i=1}^{M} P_{i}=\sum_{i=1}^{M} G P \psi_{i}\left(r\left(\mathbf{r}_{i}\right)\right)^{-4}
$$

The maximal bandwidth efficiency of a system without power equalization is then

$$
\frac{C}{W}=\log \left(1+\frac{P_{T}}{N_{0} W}\right)
$$

$C / W$ is a random variable since it is a function of the random variable $P_{T}$. One could in principle evaluate

$$
\overline{\left(\frac{C}{W}\right)}=\mathrm{E}\left[\log \left(1+\frac{P_{T}}{N_{0} W}\right)\right]
$$


However, if we think of $P_{T}$ as being obtained from an increasing number $M$ of users which scale their power so that $E\left[P_{T}\right]$ is constant for every $M$, then we can invoke the law of large numbers which tells us that $P_{T}$ converges to $E\left[P_{T}\right]$ as the number of users $M$ increases. Therefore

$$
\left(\frac{C}{W}\right) \rightarrow \log \left(1+\frac{\mathrm{E}\left[P_{T}\right]}{N_{0} W}\right)
$$

(With probability 1). The expected value of $P_{i}$ in (6) is

$$
\mathrm{E}\left[P_{i}\right]=G P \mathrm{E}\left[\psi_{i}\right] \mathrm{E}\left[\left(r\left(\mathbf{r}_{i}\right)^{-4}\right]=G P \alpha_{i} \mathrm{E}\left[\left(r\left(\mathbf{r}_{i}\right)^{-4}\right]=G P \alpha_{i} \int_{A} p_{i}\left(\mathbf{r}_{i}\right)\left(r\left(\mathbf{r}_{i}\right)\right)^{-4} \mathrm{~d} \mathbf{r}_{i},\right.\right.
$$

where we used the fact that $\psi_{i}$ and $r_{i}$ are independent. If $p_{i}(\mathbf{r})=1 /|A|$, for $i=1,2, \cdots, M$, i.e., users are uniformly distributed in the area $A$, we can further simplify (11) to obtain

$$
\mathrm{E}\left[P_{i}\right]=\alpha_{i} \frac{G P}{|A|} \int_{A}\left(r\left(\mathbf{r}_{i}\right)\right)^{-4} \mathrm{~d} \mathbf{r}_{i} .
$$

Using (12), the expected value of the total received power $P_{T}$ with $M$ users uniformly distributed over the area $A$ is

$$
\begin{aligned}
\mathrm{E}\left[P_{T}\right]=\sum_{i=1}^{M} \mathrm{E}\left[P_{i}\right] & =\sum_{i=1}^{M} \frac{G P}{|A|} \alpha_{i} \int_{A}\left(r\left(\mathbf{r}_{i}\right)\right)^{-4} \mathrm{~d} \mathbf{r}_{i} \\
& =\frac{G P}{|A|}\left(\sum_{i=1}^{M} \alpha_{i}\right) \int_{A}(r(\mathbf{r}))^{-4} \mathrm{~d} \mathbf{r} \\
& =\rho \int_{A}(r(\mathbf{r}))^{-4} \mathrm{~d} \mathbf{r}
\end{aligned}
$$

where

$$
\rho \triangleq \frac{G P}{|A|}\left(\sum_{i=1}^{M} \alpha_{i}\right)
$$

is called the average transmit power density in the cell area $A$.

For the region $A$ shown in Figure 1,

$$
\int_{A}(r(\mathbf{r}))^{-4} \mathrm{~d} \mathbf{r}=6 \int_{-\pi / 6}^{\pi / 6} \int_{\sqrt{3} R_{i} / 2 \cos \theta}^{\sqrt{3} R_{o} / 2 \cos \theta} \frac{1}{x^{4}} x d x d \theta=6\left(\frac{2 \pi}{3}+\sqrt{3}\right) \frac{R_{o}^{2}-R_{i}^{2}}{R_{o}^{2} R_{i}^{2}} .
$$

If $\alpha_{i}=1$ for all $i$, i.e., all the users transmit all the time, (14) becomes

$$
\rho=\frac{M G P}{|A|}=\frac{2 M G P}{3 \sqrt{3}\left(R_{o}^{2}-R_{i}^{2}\right)} .
$$


Hence,

$$
\begin{aligned}
\mathrm{E}\left[P_{T}\right] & =6\left(\frac{2 \pi}{3}+\sqrt{3}\right) \frac{R_{o}^{2}-R_{i}^{2}}{R_{o}^{2} R_{i}^{2}} \frac{2 M G P}{3 \sqrt{3}\left(R_{o}^{2}-R_{i}^{2}\right)} \\
& =\frac{8 \pi \sqrt{3}+36}{3^{2}} \frac{M G P}{R_{o}^{2} R_{i}^{2}} \\
& =\frac{8 \pi \sqrt{3}+36}{3^{2}} \frac{R_{o}^{2}}{R_{i}^{2}} \frac{M G P}{R_{o}^{4}} \\
& =\frac{8 \pi \sqrt{3}+36}{3^{2}} \eta^{2} P_{T_{c}}=8.84 \eta^{2} P_{T_{c}}
\end{aligned}
$$

where $\eta=R_{o} / R_{i}$ is the ratio between the outer and inner radii of the cell, and $P_{T_{c}}$, defined in (2), is the total received power of a system using power equalization and not considering shadowing effect.

Substituting $\mathrm{E}\left[P_{T}\right]=1.47 \eta^{2} P_{T_{c}}$ into (10), we obtain

$$
\left(\frac{C}{W}\right) \rightarrow \log \left(1+\frac{\mathrm{E}\left[P_{T}\right]}{N_{0} W}\right)=\log \left(1+8.84 \eta^{2} \frac{P_{T_{c}}}{N_{0} W}\right)
$$

which holds for a single-cell system without power equalization and without shadowing. The right side of (18) is plotted in Figure 2 for $\eta=100$.

\subsection{Without Power Equalization But With Shadowing}

When shadowing effects are considered, the path loss from user $i$ to the base station is no longer a function of distance. According to a widely accepted model [12], the path loss from user $i$ to the base station is given by $G 10^{\left(\xi\left(\mathbf{r}_{i}\right) / 10\right)}\left(r\left(\mathbf{r}_{i}\right)\right)^{-4}$, where $\xi\left(\mathbf{r}_{i}\right)$ is a zero-mean Gaussian random variable of standard deviation $\sigma=8(d B)$.

As a function of $\mathbf{r}, \xi(\mathbf{r})$ is a random process. Based on the experimental evidence that blockage statistics vary quite rapidly in all directions, we follow the approach used in [14] and take the spatial autocorrelation functions of $\xi(\mathbf{r})$ to be extremely narrow in all directions. That is, we assume

$$
\mathrm{E}\left[\xi(\mathbf{r}) \xi\left(\mathbf{r}^{\prime}\right)\right]=\sigma^{2} \delta\left(\mathbf{r}-\mathbf{r}^{\prime}\right),
$$

where $\delta\left(\mathbf{r}-\mathbf{r}^{\prime}\right)=0$ if $\mathbf{r} \neq \mathbf{r}^{\prime}$ and 1 otherwise. This also makes the random process $\xi(\mathbf{r})$ wide-sense stationary.

Except for the difference in path loss, the following derivations are similar to the ones in the previous section. At the base station, the received power from user $i$ at location $\mathbf{r}_{i}$ is given by

$$
P_{i}=G P \psi_{i} 10^{\xi\left(\mathbf{r}_{i}\right) / 10}\left(r\left(\mathbf{r}_{i}\right)\right)^{-4},
$$


and the total power received at the base station from all users is

$$
P_{T}=\sum_{i=1}^{M} P_{i}=\sum_{i=1}^{M} G P \psi_{i} 10^{\xi\left(\mathbf{r}_{i}\right) / 10}\left(r\left(\mathbf{r}_{i}\right)\right)^{-4} .
$$

Since the voice activity $\psi_{i}$ is independent of the user's location,

$$
\begin{aligned}
\mathrm{E}\left[P_{i}\right] & =G P \mathrm{E}\left[\psi_{i}\right] \mathrm{E}\left[10^{\xi\left(\mathbf{r}_{i}\right) / 10}\left(r\left(\mathbf{r}_{i}\right)\right)^{-4}\right] \\
& =G P \alpha_{i} \mathrm{E}\left[10^{\xi\left(\mathbf{r}_{i}\right) / 10}\left(r\left(\mathbf{r}_{i}\right)\right)^{-4}\right] \\
& =G P \alpha_{i} \mathrm{E}\left[\mathrm{E}\left[10^{\xi\left(\mathbf{r}_{i}\right) / 10} \mid \mathbf{r}_{i}\right]\left(r\left(\mathbf{r}_{i}\right)\right)^{-4}\right] \\
& =G P \alpha_{i} \int_{A} p_{i}\left(\mathbf{r}_{i}\right)\left(\int_{-\infty}^{\infty} q\left(\xi \mid \mathbf{r}_{i}\right) 10^{\xi / 10} \mathrm{~d} \xi\right)\left(r\left(\mathbf{r}_{i}\right)\right)^{-4} \mathrm{~d} \mathbf{r}_{i},
\end{aligned}
$$

where $q(\xi \mid \mathbf{r})$ is the probability density function of $\xi(\mathbf{r})$ given $\mathbf{r}$.

When users are uniformly distributed in the area $A$, we have $p_{i}(\mathbf{r})=1 /|A|$, for $i=1,2, \cdots, M$. Also, $q(\xi \mid \mathbf{r})$ is independent of $\mathbf{r}$. Hence

$$
q(\xi \mid \mathbf{r})=\frac{1}{\sqrt{2 \pi} \sigma^{2}} e^{-\xi^{2} / 2} .
$$

Then,

$$
\begin{aligned}
\mathrm{E}\left[P_{i}\right] & =G P \alpha_{i} \int_{A} \frac{1}{|A|}\left(\int_{-\infty}^{\infty} \frac{1}{\sqrt{2 \pi} \sigma} e^{-\xi^{2} / 2} 10^{\xi / 10} \mathrm{~d} \xi\right)\left(r\left(\mathbf{r}_{i}\right)\right)^{-4} \mathrm{~d} \mathbf{r}_{i} . \\
& =\frac{G P}{|A|} \alpha_{i} \int_{A}\left(\int_{-\infty}^{\infty} \frac{1}{\sqrt{2 \pi} \sigma} e^{-\xi^{2} / 2} 10^{\xi / 10} \mathrm{~d} \xi\right)\left(r\left(\mathbf{r}_{i}\right)\right)^{-4} \mathrm{~d} \mathbf{r}_{i} . \\
& =\frac{G P}{|A|} \alpha_{i} \int_{A} e^{B^{2} / 2}\left(r\left(\mathbf{r}_{i}\right)\right)^{-4} \mathrm{~d} \mathbf{r}_{i} \\
& =\frac{G P}{|A|} \alpha_{i} e^{B^{2} / 2} \int_{A}\left(r\left(\mathbf{r}_{i}\right)\right)^{-4} \mathrm{~d} \mathbf{r}_{i}
\end{aligned}
$$

where

$$
B \triangleq \sigma \ln 10 / 10 .
$$

From (20) we derive the expected value of the total average received power at the base station with $M$ users uniformly distributed over the area $A$ as

$$
\begin{aligned}
\mathrm{E}\left[P_{T}\right]=\sum_{i=1}^{M} \mathrm{E}\left[P_{i}\right] & =\frac{G P}{|A|} \sum_{i=1}^{M} \alpha_{i} e^{B^{2} / 2} \int_{A}\left(r\left(\mathbf{r}_{i}\right)\right)^{-4} \mathrm{~d} \mathbf{r}_{i} \\
& =\frac{G P}{|A|}\left(\sum_{i=1}^{M} \alpha_{i}\right) e^{B^{2} / 2} \int_{A}(r(\mathbf{r}))^{-4} \mathrm{~d} \mathbf{r} \\
& =\rho e^{B^{2} / 2} \int_{A}(r(\mathbf{r}))^{-4} \mathrm{~d} \mathbf{r} .
\end{aligned}
$$




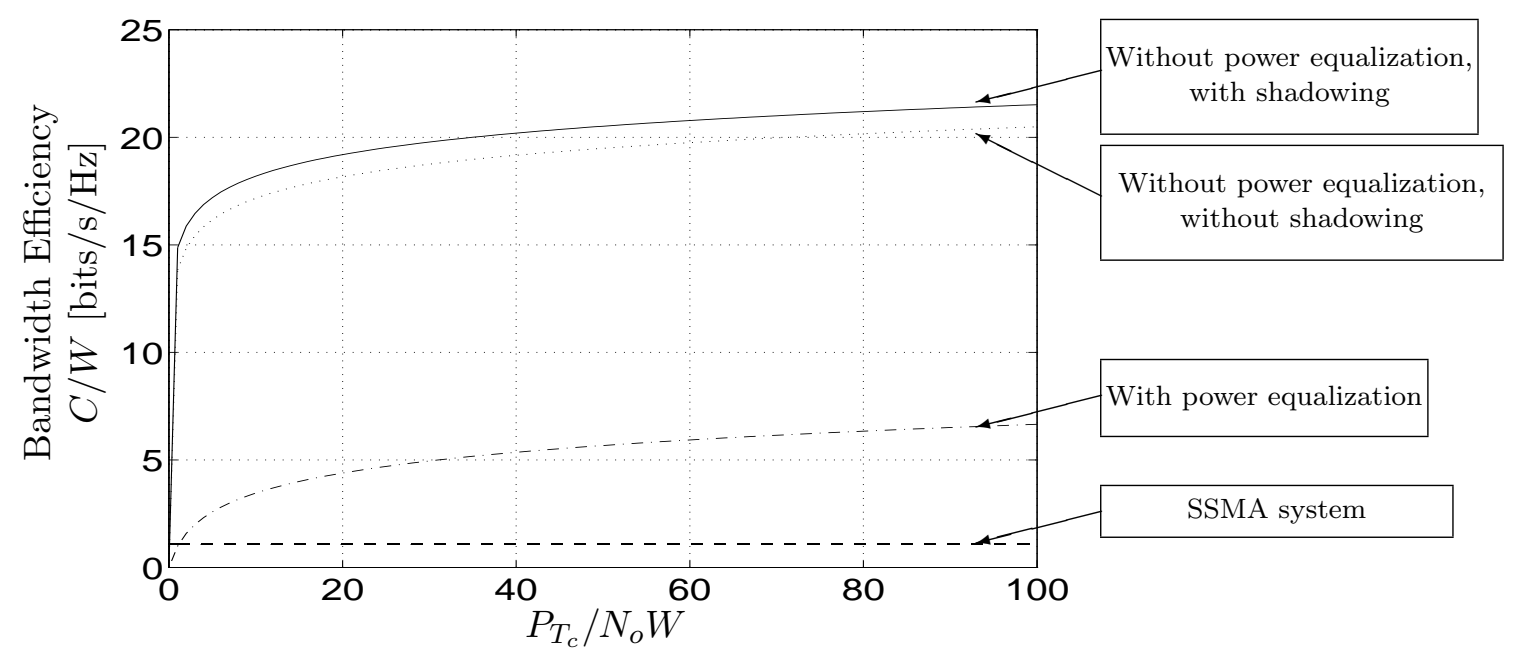

Figure 2: Bandwidth efficiency $C / W$ versus $P_{T_{c}} / N_{0} W$ for a single cell with $\eta=100$.

Assuming the cell region $A$ shown in Figure 1,

$$
\mathrm{E}\left[P_{T}\right]=\rho e^{B^{2} / 2} \int_{A}\left(r\left(\mathbf{r}_{i}\right)\right)^{-4} \mathrm{~d} \mathbf{r}_{i}=6\left(\frac{2 \pi}{3}+\sqrt{3}\right) \rho e^{\frac{B^{2}}{2}} \frac{R_{o}^{2}-R_{i}^{2}}{R_{o}^{2} R_{i}^{2}} .
$$

Using (16),

$$
\begin{aligned}
\mathrm{E}\left[P_{T}\right] & =6\left(\frac{2 \pi}{3}+\sqrt{3}\right) e^{\frac{B^{2}}{2}} \frac{2 M G P}{3 \sqrt{3}\left(R_{o}^{2}-R_{i}^{2}\right)} \frac{R_{o}^{2}-R_{i}^{2}}{R_{o}^{2} R_{i}^{2}} \\
& =\frac{8 \pi \sqrt{3}+36}{3^{2}} e^{\frac{B^{2}}{2}} \frac{R_{o}^{2}}{R_{i}^{2}} \frac{M G P}{R_{o}^{4}} \\
& =\frac{4 \pi \sqrt{3}+18}{3^{2}} e^{\frac{B^{2}}{2}} \eta^{2} P_{T_{c}}=8.84 e^{B^{2} / 2} \eta^{2} P_{T_{c}}
\end{aligned}
$$

where $\eta=R_{o} / R_{i}$ and $P_{T_{c}}$ is defined in (2). Inserting $\mathrm{E}\left[P_{T}\right]$ (26) into (10) we obtain

$$
\left(\frac{C}{W}\right) \rightarrow \log \left(1+\frac{\mathrm{E}\left[P_{T}\right]}{N_{0} W}\right)=\log \left(1+8.84 e^{B^{2} / 2} \eta^{2} \frac{P_{T_{c}}}{N_{0} W}\right),
$$

which is the largest possible bandwidth efficiency for a single-cell system without power equalization but with shadowing.

Figure 2 shows the bandwidth efficiencies of the single-cell systems that we have studied in this section. From this figure we see that the bandwidth efficiency of a single-cell system increases significantly if we do not force power equalization. This improvement is due to the fact that a mobile user with a strong path to the base station can transmit information at a higher rate instead of reducing its power. 


\section{Multi-cell system}

Now, we extend our analysis to multi-cell systems. Again, we study bandwidth efficiencies of systems a) with power equalization, b) without power equalization and without shadowing, and c) without power equalization but with shadowing.

We consider the multi-cell system that consists of an infinite number of hexagonal cells in a twodimensional plane (see Figure. 3). Each cell and its base station located at the cell center are indexed by an integer $k, k=0,1,2, \cdots$. The radius of each hexagonal cell is $R_{o}$. Without loss of generality, we consider the bandwidth efficiency of cell 0 . Let $D$ be a disk in the two-dimensional plane (see the shaded region in Figure 3). The center of the disk is base station 0. Its inner boundary is an hexagon with radius $R_{i}$, and the radius of its outer boundary is chosen sufficiently large (for instance, larger than $10 R_{o}$ ). Assume that there are $M$ users scattered in $D$. Each user is indexed by the integer $i$, $i=1,2, \cdots, M$, and the location of user $i$ in $D$ is denoted by the vector $\mathbf{r}_{i}$. We assume that the locations $\mathbf{r}_{i}$ are mutually independent random variables with identical distributions.

In the multi-cell system, we assume that a user always communicates with its electronically closest base station. That is, a user belongs to the cell whose base station receives this user with the strongest signal.

\subsection{With Power Equalization}

Consider cell 0 in the multi-cell system with power equalization. The received power of a signal user that belongs to cell 0 contributes to the signal power, whereas the received power of a user that does not belong to cell 0 contributes to the interference. Let $P_{\text {in }}$ be the total received signal power and let $I_{\text {out }}$ denote the total interference. The interference correction factor $f$ is defined as the ratio $I_{\text {out }} / P_{\text {in }}$.

We assume that the background noise $N_{0} W$ is negligible compared to the total interference $I_{\text {out }}$. The highest possible bandwidth efficiency is given by

$$
\frac{C}{W}=\log \left(1+\frac{P_{\text {in }}}{I_{\text {out }}}\right)=\log \left(1+\frac{1}{f}\right)[\mathrm{bits} / \mathrm{s} / \mathrm{Hz}] .
$$

Let $M_{0}$ denote the number of users that belong to cell 0 . Due to power equalization the received signal power of one user in cell 0 is $P_{i n} / M_{0}$. For an SSMA system that decodes each user while treating other users as noise, the "noise" seen by the user is

$$
P_{\text {in }}-\frac{P_{\text {in }}}{M_{0}}+I_{\text {out }}=P_{\text {in }}\left(1-\frac{1}{M_{0}}+f\right) .
$$




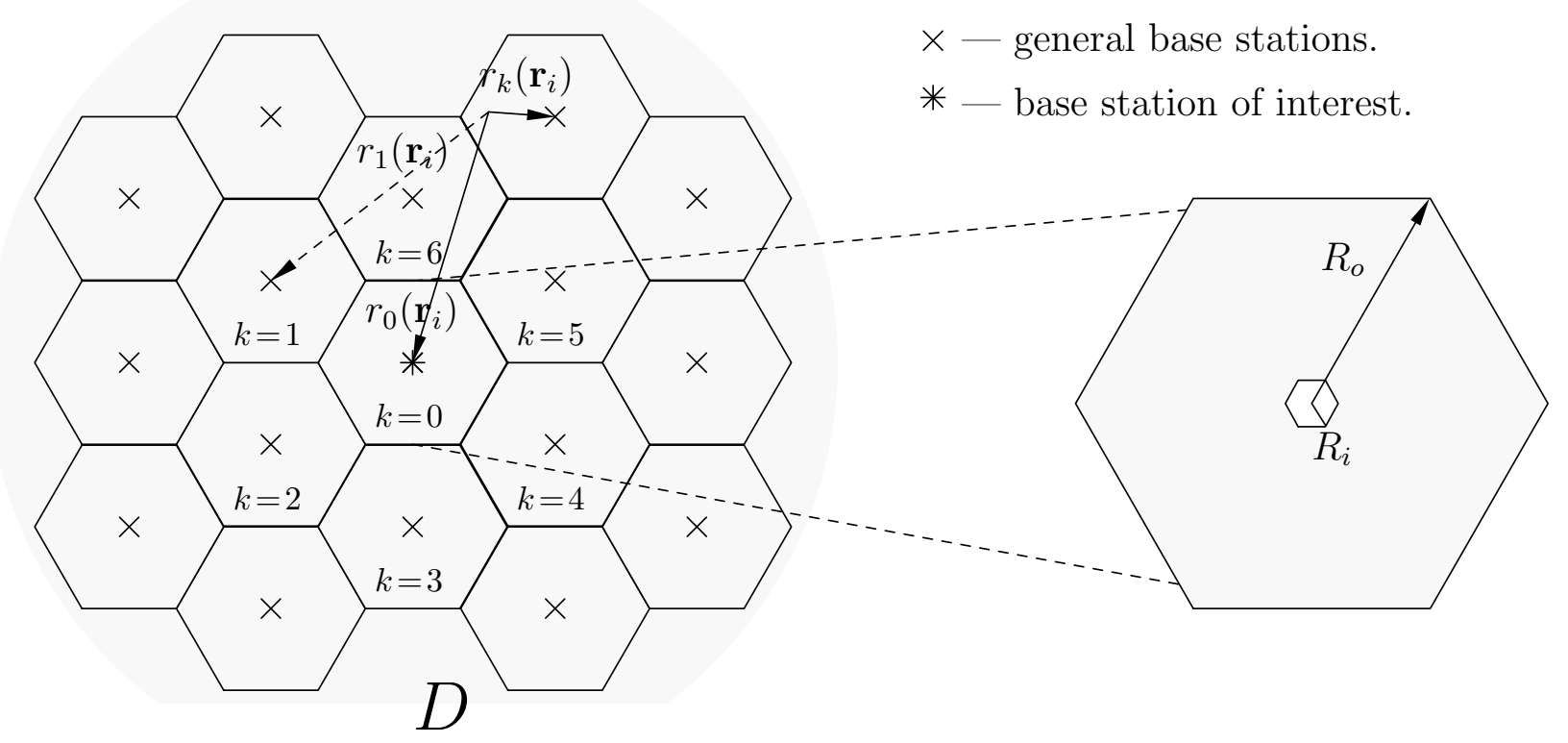

Figure 3: A finite area in the two-dimensional plane.

Therefore, the bandwidth efficiency of an SSMA system is given by

$$
\frac{C}{W}=M_{0} \log \left(1+\frac{P_{i n} / M_{0}}{P_{\text {in }}\left(1-1 / M_{0}+f\right)}\right)=M_{0} \log \left(1+\frac{1}{\left(M_{0}-1+M_{0} f\right)}\right)[\mathrm{bits} / \mathrm{s} / \mathrm{Hz}] .
$$

The value of the interference correction factor $f$ depends on the signal path loss and the distribution of users in the system. For instance, assuming that users are uniformly distributed in the region $D$ and the path loss from user $i$ to the $k$ th base station is proportional to $\left(r_{k}\left(\mathbf{r}_{i}\right)\right)^{-4}$ (i.e., there are no shadowing effects) the interference correction factor $f$ is [15]

$$
f=\frac{I_{\text {out }}}{P_{\text {in }}} \approx 0.33
$$

Given that $f=0.33$, the highest possible bandwidth efficiency of a multi-cell system with power equalization is

$$
\frac{C}{W}=\log \left(1+\frac{1}{f}\right) \approx 2.0[\mathrm{bits} / \mathrm{s} / \mathrm{Hz}] .
$$

Given that $f=0.33$ and as $M_{0} \rightarrow \infty$, the bandwidth efficiency of a multi-cell SSMA system becomes

$$
\frac{C}{W}=\frac{1}{1+f} \log e=1.08[\mathrm{bits} / \mathrm{s} / \mathrm{Hz}]
$$

With shadowing, the interference correction factor $f$ becomes larger [16]. Consequently, the bandwidth efficiency in (30) and (31) will become smaller. 


\subsection{Without Power Equalization And Without Shadowing}

Without shadowing, the signal propagation loss depends only on the distance. Let $r_{k}\left(\mathbf{r}_{i}\right)$ denote the distance from user $i$ to the $k$ th base station. Assuming fourth-power propagation loss, the path loss from user user $i$ to the $k$ th base station is proportional to $\left(r_{k}\left(\mathbf{r}_{i}\right)\right)^{-4}$. Hence, user $i$ located at $\mathbf{r}_{i}$ belongs to the $k$ th cell if the $k$ th base station is the closest to the user.

Assume that all users transmit with power $P$. At base station 0 the received power from user $i$ is given by

$$
P_{i}=G P \psi_{i}\left(r_{0}\left(\mathbf{r}_{i}\right)\right)^{-4}
$$

The total signal power $P_{i n}$ is

$$
P_{\text {in }}=\sum_{i=1}^{M} P_{i} \varnothing\left(\mathbf{r}_{i}\right)=\sum_{i=1}^{M} G P \psi_{i}\left(r_{0}\left(\mathbf{r}_{i}\right)\right)^{-4} \varnothing\left(\mathbf{r}_{i}\right),
$$

and the total interference $I_{\text {out }}$ is

$$
I_{\text {out }}=\sum_{i=1}^{M} P_{i}\left(1-\varnothing\left(\mathbf{r}_{i}\right)\right)=\sum_{i=1}^{M} G P \psi_{i}\left(r_{0}\left(\mathbf{r}_{i}\right)\right)^{-4}\left(1-\varnothing\left(\mathbf{r}_{i}\right)\right) .
$$

where the $\varnothing(\mathbf{r})$ is an indicator function telling whether or not a user at location $\mathbf{r}$ belongs to cell 0 . Without shadowing, the indicator function is defined as

$$
\varnothing(\mathbf{r})= \begin{cases}1, & \text { if } r_{0}(\mathbf{r})=\min _{\forall k^{\prime}} r_{k^{\prime}}(\mathbf{r}) \\ 0, & \text { otherwise. }\end{cases}
$$

Since voice activities $\psi_{i}$ and locations $\mathbf{r}_{i}$ are mutually independent random variables, the received powers $P_{i}$ are mutually independent. Hence, the total signal power $P_{\text {in }}$ and $I_{\text {out }}$ are sums of independent random variables, and the bandwidth efficiency of the system without power equalization is the random variable

$$
\frac{C}{W}=\log \left(1+\frac{P_{\text {in }}}{I_{\text {out }}}\right)
$$

Again,

$$
\left(\frac{C}{W}\right) \rightarrow \log \left(1+\frac{\mathrm{E}\left[P_{\text {in }}\right]}{\mathrm{E}\left[I_{\text {out }}\right]}\right)
$$

(with probability 1). 
The expected value of $P_{\text {in }}$ in (33) can be derived as

$$
\begin{aligned}
\mathrm{E}\left[P_{i n}\right] & =\mathrm{E}\left[\sum_{i=1}^{M} G P \psi_{i}\left(r_{0}\left(\mathbf{r}_{i}\right)\right)^{-4} \varnothing\left(\mathbf{r}_{i}\right)\right] \\
& =G P \sum_{i=1}^{M} \mathrm{E}\left[\psi_{i}\right] \mathrm{E}\left[\left(r_{0}\left(\mathbf{r}_{i}\right)\right)^{-4} \varnothing\left(\mathbf{r}_{i}\right)\right] \\
& =G P \sum_{i=1}^{M} \alpha_{i} \int_{D} p_{i}(\mathbf{r})\left(r_{0}(\mathbf{r})\right)^{-4} \varnothing(\mathbf{r}) \mathrm{d} \mathbf{r} .
\end{aligned}
$$

Assuming uniformly distributed users in $D$,

$$
\begin{aligned}
\mathrm{E}\left[P_{i n}\right] & =G P \sum_{i=1}^{M} \alpha_{i} \int_{D} \frac{1}{|D|}\left(r_{0}(\mathbf{r})\right)^{-4} \emptyset(\mathbf{r}) \mathrm{d} \mathbf{r} \\
& =\frac{G P}{|D|}\left(\sum_{i=1}^{M} \alpha_{i}\right) \int_{D}\left(r_{0}(\mathbf{r})\right)^{-4} \emptyset(\mathbf{r}) \mathrm{d} \mathbf{r} \\
& =\rho \int_{D}\left(r_{0}(\mathbf{r})\right)^{-4} \emptyset(\mathbf{r}) \mathrm{d} \mathbf{r},
\end{aligned}
$$

where $\rho$ is defined in (14).

Similarly, the expected value of $I_{\text {out }}$ in (34) can be derived as

$$
\begin{aligned}
\mathrm{E}\left[I_{\text {out }}\right] & =\mathrm{E}\left[\sum_{i=1}^{M} G P \psi_{i}\left(r_{0}\left(\mathbf{r}_{i}\right)\right)^{-4}\left(1-\varnothing\left(\mathbf{r}_{i}\right)\right)\right] \\
& =G P \sum_{i=1}^{M} \mathrm{E}\left[\psi_{i}\right] \mathrm{E}\left[\left(r_{0}\left(\mathbf{r}_{i}\right)\right)^{-4}\left(1-\varnothing\left(\mathbf{r}_{i}\right)\right)\right] \\
& =G P \sum_{i=1}^{M} \alpha_{i} \int_{D} p_{i}(\mathbf{r})\left(r_{0}(\mathbf{r})\right)^{-4}(1-\varnothing(\mathbf{r})) \mathrm{d} \mathbf{r} .
\end{aligned}
$$

With $p_{i}(\mathbf{r})=1 /|D|$ for all $i,(40)$ becomes

$$
\begin{aligned}
\mathrm{E}\left[I_{\text {out }}\right] & =G P \sum_{i=1}^{M} \alpha_{i} \int_{D} \frac{1}{|D|}\left(r_{0}(\mathbf{r})\right)^{-4}(1-\varnothing(\mathbf{r})) \mathrm{d} \mathbf{r} \\
& =\frac{G P}{|D|}\left(\sum_{i=1}^{M} \alpha_{i}\right) \int_{D}\left(r_{0}(\mathbf{r})\right)^{-4}(1-\varnothing(\mathbf{r})) \mathrm{d} \mathbf{r} \\
& =\rho \int_{D}\left(r_{0}(\mathbf{r})\right)^{-4}(1-\varnothing(\mathbf{r})) \mathrm{d} \mathbf{r} .
\end{aligned}
$$

Given the indicator function in (35) and the cell deployment in Figure 3, the integral in (39) can be computed as

$$
\int_{D}\left(r_{0}(\mathbf{r})\right)^{-4} \varnothing(\mathbf{r}) \mathrm{d} \mathbf{r}=6 \int_{-\pi / 6}^{\pi / 6} \int_{\sqrt{3} R_{i} / 2 \cos \theta}^{\sqrt{3} R_{o} / 2 \cos \theta} \frac{1}{x^{4}} x d x d \theta=6\left(\frac{2 \pi}{3}+\sqrt{3}\right) \frac{R_{o}^{2}-R_{i}^{2}}{R_{o}^{2} R_{i}^{2}},
$$


and the integral in (41) becomes

$$
\int_{D}\left(r_{0}(\mathbf{r})\right)^{-4}(1-\varnothing(\mathbf{r})) \mathrm{d} \mathbf{r}=6 \int_{-\pi / 6}^{\pi / 6} \int_{\sqrt{3} R_{o} / 2 \cos \theta}^{\infty} \frac{1}{x^{4}} x d x d \theta=6\left(\frac{2 \pi}{3}+\sqrt{3}\right) \frac{1}{R_{o}^{2}} .
$$

Inserting (42) and (43) into (39) and (41), respectively, we obtain

$$
\mathrm{E}\left[P_{i n}\right]=6 \rho\left(\frac{2 \pi}{3}+\sqrt{3}\right) \frac{R_{o}^{2}-R_{i}^{2}}{R_{o}^{2} R_{i}^{2}}
$$

and

$$
\mathrm{E}\left[I_{\text {out }}\right]=6 \rho\left(\frac{2 \pi}{3}+\sqrt{3}\right) \frac{1}{R_{o}^{2}} .
$$

Using (44) and (45) into (37), we obtain the average bandwidth efficiency of the multi-cell system without power equalization and without shadowing, namely

$$
\left(\frac{C}{W}\right) \rightarrow \log \left(1+\frac{R_{o}^{2}-R_{i}^{2}}{R_{i}^{2}}\right)=\log \left(\eta^{2}\right)[\mathrm{bits} / \mathrm{s} / \mathrm{Hz}],
$$

where $\eta=R_{o} / R_{i}$.

\subsection{Without Power Equalization But With Shadowing}

When shadowing is considered, the path loss from user $i$ to the $k$ th base station is proportional to $10^{\left(\xi_{k}\left(\mathbf{r}_{i}\right) / 10\right)}\left(r_{k}\left(\mathbf{r}_{i}\right)\right)^{-4}[12]$, where $\xi_{k}\left(\mathbf{r}_{i}\right)$, for the given $\mathbf{r}_{i}$, is a zero mean Gaussian random variable with standard deviation $\sigma=8(\mathrm{~dB})$.

$\xi_{k}(\mathbf{r})$ can be seen as a random process for each $k$. Following the approach of [14] we take the spatial autocorrelation functions of $\xi_{k}(\mathbf{r})$ to be extremely narrow in all directions for each $k$. That is, we assume

$$
E\left[\xi_{k}(\mathbf{r}) \xi_{k}\left(\mathbf{r}^{\prime}\right)\right]=\sigma^{2} \delta\left(\mathbf{r}-\mathbf{r}^{\prime}\right), \quad \forall k .
$$

Also, we take the random processes $\xi_{k}\left(\mathbf{r}_{i}\right)$ and $\xi_{k^{\prime}}\left(\mathbf{r}_{i}\right)$ to be mutually independent when $k \neq k^{\prime}$ because they are associated with the two different paths.

Again, assume all users transmit with power $P$ since there is no power equalization. At base station 0 the received power from user $i$ is given by

$$
P_{i}=G P \psi_{i} 10^{\xi_{0}\left(\mathbf{r}_{i}\right) / 10}\left(r_{0}\left(\mathbf{r}_{i}\right)\right)^{-4}
$$


The total signal power $P_{i n}$ is

$$
P_{i n}=\sum_{i=1}^{M} P_{i} \varnothing\left(\mathbf{r}_{i}\right)=\sum_{i=1}^{M} G P \psi_{i} 10^{\xi_{0}\left(\mathbf{r}_{i}\right) / 10}\left(r_{0}\left(\mathbf{r}_{i}\right)\right)^{-4} \varnothing\left(\mathbf{r}_{i}\right),
$$

and the total interference $I_{\text {out }}$ is

$$
I_{\text {out }}=\sum_{i=1}^{M} P_{i}\left(1-\varnothing\left(\mathbf{r}_{i}\right)\right)=\sum_{i=1}^{M} G P \psi_{i} 10^{\xi_{0}\left(\mathbf{r}_{i}\right) / 10}\left(r_{0}\left(\mathbf{r}_{i}\right)\right)^{-4}\left(1-\varnothing\left(\mathbf{r}_{i}\right)\right),
$$

where the $\varnothing(\mathbf{r})$ is an indicator function telling whether or not a user at location $\mathbf{r}$ belongs to cell 0 . Since shadowing is considered, the indicator function here is different from that in (35) and is defined as

$$
\varnothing\left(\mathbf{r}_{i}\right)= \begin{cases}1, & \text { if } 10^{-\xi_{0}(\mathbf{r}) / 10}\left(r_{0}(\mathbf{r})\right)^{4}=\min _{k} 10^{-\xi_{k}(\mathbf{r}) / 10}\left(r_{k}(\mathbf{r})\right)^{4}, \\ 0, & \text { if } 10^{-\xi_{0}(\mathbf{r}) / 10}\left(r_{0}(\mathbf{r})\right)^{4}>\min _{k} 10^{-\xi_{k}(\mathbf{r}) / 10}\left(r_{k}(\mathbf{r})\right)^{4} .\end{cases}
$$

Since the voice activities $\psi_{i}$ and locations $\mathbf{r}_{i}$ are mutually independent random variables and given that the random processes $\xi_{k}(\mathbf{r})$ are white, the received powers $P_{i}$ are independent random variables. Hence, $P_{i n}$ and $I_{\text {out }}$ are sums of independent random variables. By the law of large number, as the number of users $M$ increases, $P_{\text {in }}$ and $I_{\text {out }}$ converge to $\mathrm{E}\left[P_{\text {in }}\right]$ and $\mathrm{E}\left[I_{\text {out }}\right]$ with probability 1 , respectively. Therefore, we can use (37) to approximately compute the average bandwidth efficiency when the number of users $M$ in the system is sufficiently large.

The expected value of $P_{i n}$ in (49) can be derived as

$$
\begin{aligned}
\mathrm{E}\left[P_{i n}\right] & =\mathrm{E}\left[\sum_{i=1}^{M} G P \psi_{i} 10^{\xi_{0}\left(\mathbf{r}_{i}\right) / 10}\left(r_{0}\left(\mathbf{r}_{i}\right)\right)^{-4} \varnothing\left(\mathbf{r}_{i}\right)\right] \\
& =G P \sum_{i=1}^{M} \mathrm{E}\left[\psi_{i}\right] \mathrm{E}\left[10^{\xi_{0}\left(\mathbf{r}_{i}\right) / 10}\left(r_{0}\left(\mathbf{r}_{i}\right)\right)^{-4} \varnothing\left(\mathbf{r}_{i}\right)\right] \\
& =G P \sum_{i=1}^{M} \alpha_{i} \mathrm{E}\left[\mathrm{E}\left[10^{\xi_{0}\left(\mathbf{r}_{i}\right) / 10} \emptyset\left(\mathbf{r}_{i}\right) \mid \mathbf{r}_{i}\right]\left(r_{0}\left(\mathbf{r}_{i}\right)\right)^{-4}\right] \\
& =G P \sum_{i=1}^{M} \alpha_{i} \int_{D} p_{i}(\mathbf{r}) \mathrm{E}\left[10^{\xi_{0}(\mathbf{r}) / 10} \varnothing(\mathbf{r}) \mid \mathbf{r}\right]\left(r_{0}(\mathbf{r})\right)^{-4} \mathrm{~d} \mathbf{r} .
\end{aligned}
$$


Similarly,

$$
\begin{aligned}
\mathrm{E}\left[I_{\text {out }}\right] & =\mathrm{E}\left[\sum_{i=1}^{M} G P \psi_{i} 10^{\xi_{0}\left(\mathbf{r}_{i}\right) / 10}\left(r_{0}\left(\mathbf{r}_{i}\right)\right)^{-4}\left(1-\varnothing\left(\mathbf{r}_{i}\right)\right)\right] \\
& =G P \sum_{i=1}^{M} \mathrm{E}\left[\psi_{i}\right] \mathrm{E}\left[10^{\xi_{0}\left(\mathbf{r}_{i}\right) / 10}\left(r_{0}\left(\mathbf{r}_{i}\right)\right)^{-4}\left(1-\varnothing\left(\mathbf{r}_{i}\right)\right)\right] \\
& =G P \sum_{i=1}^{M} \alpha_{i} \mathrm{E}\left[\mathrm{E}\left[10^{\xi_{0}\left(\mathbf{r}_{i}\right) / 10}\left(1-\varnothing\left(\mathbf{r}_{i}\right)\right) \mid \mathbf{r}_{i}\right]\left(r_{0}\left(\mathbf{r}_{i}\right)\right)^{-4}\right] \\
& =G P \sum_{i=1}^{M} \alpha_{i} \int_{D} p_{i}(\mathbf{r}) \mathrm{E}\left[10^{\xi_{0}(\mathbf{r}) / 10}(1-\varnothing(\mathbf{r})) \mid \mathbf{r}\right]\left(r_{0}(\mathbf{r})\right)^{-4} \mathrm{~d} \mathbf{r} .
\end{aligned}
$$

When users are uniformly distributed in $D$, i.e., $p_{i}(\mathbf{r})=1 /|D|$ for all $i,(52)$ and (53) become

$$
\begin{aligned}
\mathrm{E}\left[P_{i n}\right] & =G P \sum_{i=1}^{M} \alpha_{i} \int_{D} \frac{1}{|D|} \mathrm{E}\left[10^{\xi_{0}(\mathbf{r}) / 10} \varnothing(\mathbf{r}) \mid \mathbf{r}\right]\left(r_{0}(\mathbf{r})\right)^{-4} \mathrm{~d} \mathbf{r} \\
& =\frac{G P}{|D|}\left(\sum_{i=1}^{M} \alpha_{i}\right) \int_{D} \mathrm{E}\left[10^{\xi_{0}(\mathbf{r}) / 10} \varnothing(\mathbf{r}) \mid \mathbf{r}\right]\left(r_{0}(\mathbf{r})\right)^{-4} \mathrm{~d} \mathbf{r} \\
& =\rho \int_{D} \mathrm{E}\left[10^{\xi_{0}(\mathbf{r}) / 10} \varnothing(\mathbf{r}) \mid \mathbf{r}\right]\left(r_{0}(\mathbf{r})\right)^{-4} \mathrm{~d} \mathbf{r},
\end{aligned}
$$

and

$$
\begin{aligned}
\mathrm{E}\left[I_{\text {out }}\right] & =G P \sum_{i=1}^{M} \alpha_{i} \int_{D} \frac{1}{|D|} \mathrm{E}\left[10^{\xi_{0}(\mathbf{r}) / 10}(1-\varnothing(\mathbf{r})) \mid \mathbf{r}\right]\left(r_{0}(\mathbf{r})\right)^{-4} \mathrm{~d} \mathbf{r} \\
& =\frac{G P}{|D|}\left(\sum_{i=1}^{M} \alpha_{i}\right) \int_{D} \mathrm{E}\left[10^{\xi_{0}(\mathbf{r}) / 10}(1-\varnothing(\mathbf{r})) \mid \mathbf{r}\right]\left(r_{0}(\mathbf{r})\right)^{-4} \mathrm{~d} \mathbf{r} \\
& =\rho \int_{D} \mathrm{E}\left[10^{\xi_{0}(\mathbf{r}) / 10}(1-\varnothing(\mathbf{r})) \mid \mathbf{r}\right]\left(r_{0}(\mathbf{r})\right)^{-4} \mathrm{~d} \mathbf{r} .
\end{aligned}
$$

Given the indicator function in (51), the expected value of the total signal power $P_{i n}$ in (54) can be further written as

$$
\mathrm{E}\left[P_{i n}\right]=\rho \int_{D} E\left[\varnothing(\mathbf{r}) 10^{\xi_{0}(\mathbf{r}) / 10} \mid \mathbf{r}\right]\left(r_{0}(\mathbf{r})\right)^{-4} \mathrm{~d} \mathbf{r} \triangleq \rho P_{1}\left(R_{0}, \eta, \sigma\right)
$$

where

$$
\begin{aligned}
& P_{1}\left(R_{0}, \eta, \sigma\right) \triangleq \int_{D} E\left[\varnothing(\mathbf{r}) 10^{\xi_{0}(\mathbf{r}) / 10} \mid \mathbf{r}\right]\left(r_{0}(\mathbf{r})\right)^{-4} \mathrm{~d} \mathbf{r} \\
& \quad=\int_{D} E\left[10^{\xi_{0}(\mathbf{r}) / 10} \prod_{k \neq 0}\left(1-Q\left(\xi_{0}(\mathbf{r})+40 \log _{10}\left(r_{k}(\mathbf{r}) / r_{0}(\mathbf{r})\right) / \sigma\right)\right)\right]\left(r_{0}(\mathbf{r})\right)^{-4} \mathrm{~d} \mathbf{r} .
\end{aligned}
$$




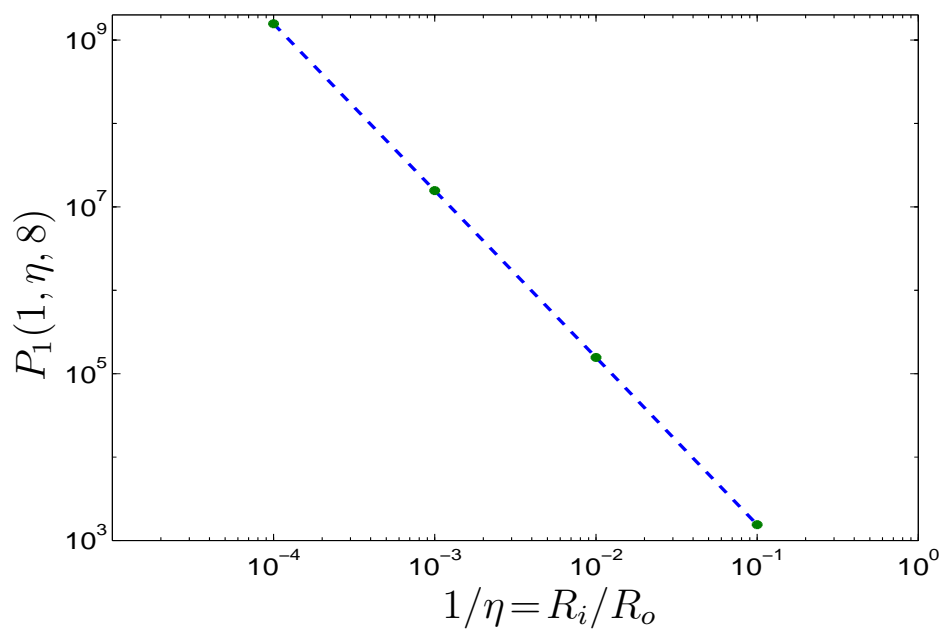

Figure 4: The numerical calculation result of $P_{1}(1, \eta, 8)$.

The detailed derivation of (57) is given in Appendix $\mathrm{A}$, and the function $Q(x)$ is defined as

$$
Q(x)=\int_{x}^{\infty} \frac{1}{\sqrt{2 \pi}} e^{-\frac{y^{2}}{2}} d y .
$$

The integral $P_{1}\left(R_{0}, \eta, \sigma\right)$ is over the two-dimensional region $D$. This integral will be evaluated numerically. Its d value depends on the region $D$, the cell radii $R_{i}$ and $R_{o}$, and the variance $\sigma$.

Notice that $D$ is a disk in the two-dimensional plane (see Figure 3), and the radius of its outer boundary is chosen large enough such that the contribution from users outside this outer boundary of $D$ to base station 0 is negligible. By normalizing the outer-radius $R_{o}$ to $R_{o}=1$ and choosing the variance $\sigma=8(\mathrm{~dB})$, we numerically calculated $P_{1}\left(R_{o}, \eta, \sigma\right)$, as shown in Figure 4 . It can be observed that $P_{1}(1, \eta, \sigma)$ increases as the ratio $\eta$ decreases. More precisely, we have (for $\eta$ within the displayed range in the figure)

$$
P_{1}(1, \eta, 8) \approx 15.60 \eta^{2}
$$

and, therefore,

$$
\left.\mathrm{E}\left[P_{i n}\right]\right|_{R_{o}=1, \sigma=8}=\rho P_{1}(1, \eta, 8) \approx 15.60 \rho \eta^{2}
$$

For $R_{o} \neq 1$, we can obtain $P_{1}\left(R_{o}, \eta, \sigma\right)$ from $P_{1}(1, \eta, 8)$ by scaling the coordinate system by the factor $1 / R_{o}$. Knowing that $R_{i}=R_{o} / \eta$, we see that

$$
P_{1}\left(R_{o}, \eta, 8\right)=P_{1}(1, \eta, 8) R_{o}^{-2} \approx 15.60\left(1 / R_{i}\right)^{2}
$$




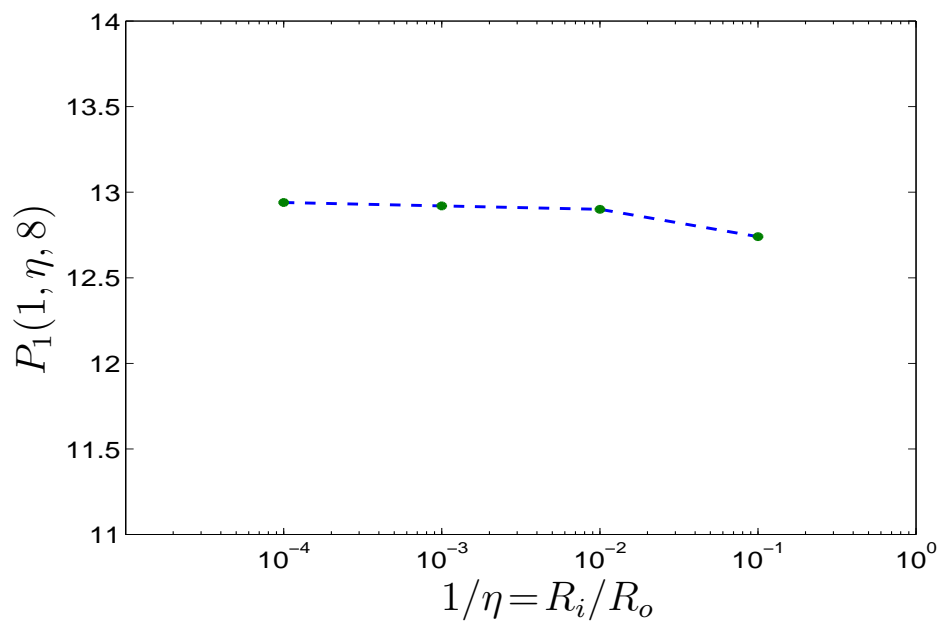

Figure 5: The numerical calculation result of $P_{1}(1, \eta, 8)$.

Inserting (58) into (56), we obtain

$$
\left.\mathrm{E}\left[P_{i n}\right]\right|_{\sigma=8}=\rho P_{1}\left(R_{o}, \eta, 8\right) \approx 15.60 \rho R_{i}^{-2}
$$

Similarly, the expected value of the total interference $I_{\text {out }}$ in $(55)$ can be written as

$$
\mathrm{E}\left[I_{\text {out }}\right]=\rho \int_{D} E\left[(1-\varnothing(\mathbf{r})) 10^{\xi_{0}(\mathbf{r}) / 10} \mid \mathbf{r}\right]\left(r_{0}(\mathbf{r})\right)^{-4} \mathrm{~d} \mathbf{r} \triangleq \rho I_{1}\left(R_{0}, \eta, \sigma\right)
$$

where

$$
\begin{aligned}
I_{1}\left(R_{0}, \eta, \sigma\right) & =\int_{D} E\left[(1-\varnothing(\mathbf{r})) 10^{\xi_{0}(\mathbf{r}) / 10} \mid \mathbf{r}\right]\left(r_{0}(\mathbf{r})\right)^{-4} \mathrm{~d} \mathbf{r} \\
& =\int_{D} E\left[10^{\xi_{0}(\mathbf{r}) / 10} \mid \mathbf{r}\right]\left(r_{0}(\mathbf{r})\right)^{-4} \mathrm{~d} \mathbf{r}-\int_{D} E\left[\varnothing(\mathbf{r}) 10^{\xi_{0}(\mathbf{r}) / 10} \mid \mathbf{r}\right]\left(r_{0}(\mathbf{r})\right)^{-4} \mathrm{~d} \mathbf{r} \\
& =e^{\frac{B^{2}}{2}} \int_{D}\left(r_{0}(\mathbf{r})\right)^{-4} \mathrm{~d} \mathbf{r}-P_{1}\left(R_{0}, \eta, \sigma\right) .
\end{aligned}
$$

$B$ is defined as $B \triangleq \sigma \ln 10 / 10$.

With the same assumption about the area $D$, we can numerically obtain $I_{1}(1, \eta, \sigma)$ and the result is shown in Figure 5. It can be seen that $I_{1}(1, \eta, \sigma) \leq 13.00$. Therefore, by scaling the coordinates we obtain

$$
I_{1}\left(R_{0}, \eta, \sigma\right) \leq 13.00 R_{o}^{-2}
$$

and from (60) we have

$$
\left.\mathrm{E}\left[I_{\text {out }}\right]\right|_{\sigma=8}=\rho I_{1}\left(R_{o}, \eta, 8\right) \leq 13.00 \rho R_{o}^{-2}
$$




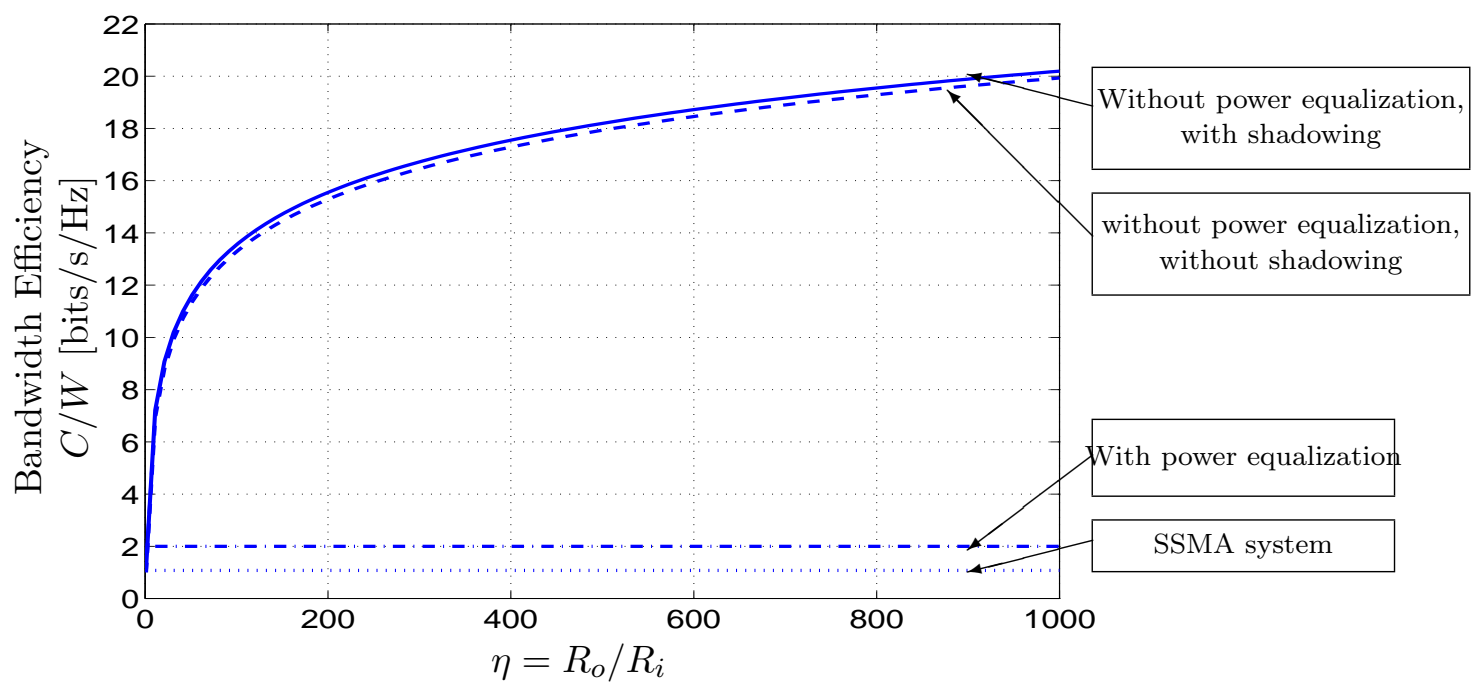

Figure 6: Bandwidth efficiency $C / W$ versus $\eta$ for multi cell systems.

Inserting (59) and (63) into (37), the largest possible bandwidth efficiency for without power equalization satisfies

$$
\left(\frac{C}{W}\right) \rightarrow \log \left(1+\frac{15.60}{13.00} \frac{R_{o}^{2}}{R_{i}^{2}}\right) \approx \log \left(1+1.20 \eta^{2}\right)[\mathrm{bits} / \mathrm{s} / \mathrm{Hz}]
$$

The bandwidth efficiencies of multi-cell systems as a functions of $\eta$ is plotted in 6 . The lowest plot represents the bandwidth efficiencies of an SSMA system (Eqn. (31)). The remaining plots are the largest possible bandwidth efficiency for a system with power equalization (Eqn. (30)), a system without power equalization and without shadowing (Eqn. (46)), and a system without power equalization but with shadowing (Eqn. (64)). We see that the conclusion is similar to that of the single-cell case considered in Section 2, namely that power equalization considerably reduces the largest possible sum-rate.

\section{Conclusion}

We have extended [11] to take into account shadowing. Also in the presence of shadowing, when the number of users is sufficiently large, there is a significant gap between the bandwidth efficiency of a cellular system that forces power equalization and one that does not. The latter is better and the improvement is mainly due to the fact that a mobile user with a strong path to the base station can transmit information at a higher rate instead of reducing its power. Such users are typically close to the base station and therefore create little interference to other cells. 
Power equalization is needed in spread spectrum multiple access with conventional matched filter detectors but it is not needed in general. In particular, successive decoding does not require power equalization.

\section{A Appendix}

\section{Derivation of the expectation in (57)}

In the following derivation, the independent variable $\mathbf{r}$ is dropped for simplicity. However, we need to keep in mind that $\varnothing, r_{0}, r_{k}, \xi_{0}$, and $\xi_{k}$ are functions of $\mathbf{r}$. Given the indicator function in (51), we have

$$
\begin{aligned}
E\left[\varnothing(\mathbf{r}) 10^{\xi_{0}(\mathbf{r}) / 10} \mid \mathbf{r}\right] & \left.=E\left[10^{\xi_{0} / 10} E\left[\varnothing \mid \xi_{0}\right)\right]\right] \\
& =E\left[10^{\xi_{0} / 10} \operatorname{Pr}\left\{\varnothing=1 \mid \xi_{0}\right\}\right] \\
& =E\left[10^{\xi_{0} / 10} \operatorname{Pr}\left\{10^{-\xi_{0} / 10} r_{0}^{4} \leq \min _{k \neq 0} 10^{-\xi_{k} / 10} r_{k}^{4} \mid \xi_{0}\right\}\right] \\
& =E\left[10^{\xi_{0} / 10} \operatorname{Pr}\left\{-\xi_{0} \leq \min _{k \neq 0}-\xi_{k}+40 \log _{10}\left(r_{k} / r_{0}\right) \mid \xi_{0}\right\}\right] \\
& =E\left[10^{\xi_{0} / 10} \prod_{k \neq 0} \operatorname{Pr}\left\{-\xi_{0} \leq-\xi_{k}+40 \log _{10}\left(r_{k} / r_{0}\right) \mid \xi_{0}\right\}\right] \\
& =E\left[10^{\xi_{0} / 10} \prod_{k \neq 0} \operatorname{Pr}\left\{\xi_{k} \leq \xi_{0}+40 \log _{10}\left(r_{k} / r_{0}\right) \mid \xi_{0}\right\}\right] \\
& =E\left[10^{\xi_{0} / 10} \prod_{k \neq 0}\left(1-Q\left(\xi_{0}+40 \log _{10}\left(r_{k} / r_{0}\right) / \sigma\right)\right)\right],
\end{aligned}
$$

where (65) is obtained due to the fact that $\xi_{k}$ 's are mutually independent, and the function $Q(x)$ is defined as

$$
Q(x)=\int_{x}^{\infty} \frac{1}{\sqrt{2 \pi}} e^{-\frac{y^{2}}{2}} d y .
$$

\section{References}

[1] Bixio Rimoldi and Rüdiger Urbanke. A rate-splitting approach to the Gaussian multiple-access channel. IEEE Trans. Inform. Theory, IT-42:364-375, March 1996.

[2] C. Berrou, A. Glavieux, and P. Thitimajshima, "Near Shannon limit error correcting coding and decoding: turbo-codes," in Proceeding of ICC '93, (Geneva), pp. 1607-1070, May 1993. 
[3] Tom Richardson and Ruediger Urbanke, "The Capacity of Low-Density Parity Check Codes under Message-Passing Decoding," preprint, Nov. 1998.

[4] R. G. Gallager, Low Density Parity-Check Codes. Cambridge, Massachusetts: M.I.T. Press, 1963.

[5] D. J. C. MacKay, "Good Error Correcting Codes based on Very Sparse Matrices." Submitted to IEEE Transactions on Information Theory. Available from http://wol.ra.phy.cam.ac.uk/, 1997

[6] Bixio Rimoldi. RDMA for multipath multiple access channels: an optimum asynchronous low complexity technique. Proc. Yth joint Swedish-Russian international workshop on information theory, pages 196-199, St. Petersburg, Russia, June 17-22, 1995.

[7] Alex Grant, Bixio Rimoldi, Rüdiger Urbanke and Phil Whiting. Rate-Splitting multiple access for discrete memoryless channels. To appear in IEEE Trans. Info. Theory.

[8] D.Tse and S. Hanly, Multi-Access Fading Channels: Part I: Polymatroid Structure, Optimal Resource Allocation and Throughput Capacities. To appear in IEEE Trans. Info. Theory.

[9] T. M. Cover and J. A. Thomas. Elements of Information Theory. Wiley, New York, 1991.

[10] Andrew J. Viterbi. Performance limits of error-correcting coding in multi-cellular CDMA systems with and without interference cancellation. In S. G. Glisic and P. A. Leppanen, editors, Code Division Multiple Access Communications, pages 47-52. Kluwer Academic Publishers, 1995.

[11] Bixio Rimoldi and Quinn Li. Potential impact of rate-splitting multiple access on cellular communications. In Proceedings of the 1996 Global Telecommunications Conference, London, Nov 18-22 1996.

[12] W. C. Y. Lee. Overview of cellular CDMA. IEEE Trans. Veh. Tech., 40:291-302, May 1991.

[13] Sergio Verdú, Multiuser Detection, Cambridge University Press, 1998.

[14] K. S. Gilhousen, I. M. Jacobs, R. Padovani, A. J. Viterbi, L. A. Weaver, and C. E. Wheatley III. On the capacity of a cellular CDMA system. IEEE Trans. Veh. Tech., 40:303-312, May 1991.

[15] K. I. Kim. CDMA cellular engineering issues. IEEE Trans. Veh. Tech., 42:345-350, August 1993. 
[16] A. J. Viterbi, A. M. Viterbi, and E. Zehavi. Other-cell interference in cellular power-controlled CDMA IEEE Trans. Comm., 42:1501-1504, April 1994. 